\title{
Retrospective and prospective assessments of gambling-related behaviors across the female menstrual cycle
}

\author{
KAYLA M. JOYCE ${ }^{1}$, AMANDA HUDSON ${ }^{2}$, ROISIN M. O'CONNOR ${ }^{3}$, ABBY L. GOLDSTEIN $^{4}$, MICHAEL ELLERY ${ }^{5}$, \\ DANIEL S. MCGRATH ${ }^{6}$, TARA S. PERROT ${ }^{7}$ and SHERRY H. STEWART ${ }^{8 *}$ \\ ${ }^{1}$ Department of Psychiatry, Dalhousie University, Halifax, Canada \\ ${ }^{2}$ Department of Psychology, University of Prince Edward Island, Charlottetown, Canada \\ ${ }^{3}$ Department of Psychology, Concordia University, Montréal, Canada \\ ${ }^{4}$ Department of Applied Psychology and Human Development, Ontario Institute for Studies in Education, University of Toronto, \\ Toronto, Canada \\ ${ }^{5}$ Department of Psychology, University of Winnipeg, Winnipeg, Canada \\ ${ }^{6}$ Department of Psychology, University of Calgary, Calgary, Canada \\ ${ }^{7}$ Department of Psychology and Neuroscience, Dalhousie University, Halifax, Canada \\ ${ }^{8}$ Departments of Psychiatry and Psychology and Neuroscience, Dalhousie University, Halifax, Canada
}

(Received: June 29, 2018; revised manuscript received: November 23, 2018; accepted: November 24, 2018)

\begin{abstract}
Background and aims: Despite increases in female gambling, little research investigates female-specific factors affecting gambling behavior (GB). Although research suggests that some addictive behaviors may fluctuate across menstrual cycle phase (MCP), gambling requires further investigation. In two studies, we examined associations between MCP and three risky GBs: time spent gambling, money spent gambling, and the probability of consuming alcohol while gambling. Associations between MCP and negative affect were also examined in Study 2. We predicted that, consistent with self-medication theory, increases in negative affect (Study 2) and risky GBs (Studies 1 and 2) would occur premenstrually/menstrually relative to other phases. Methods: Data were obtained from 33 female gamblers using a retrospective timeline followback procedure (Study 1) and from 20 female gamblers using a prospective 32-day, daily diary method (Study 2). In Study 2, salivary progesterone levels verified self-reported MCP validity. Results: Findings revealed significant, but somewhat inconsistent, MCP effects on GBs across studies. The self-medication hypothesis was partially supported. Increases relative to another $\mathrm{MCP}(\mathrm{s})$ were found for alcohol consumption while gambling premenstrually, time spent gambling menstrually/premenstrually, money spent gambling menstrually, and negative affect premenstrually. Unexpectedly, findings more consistently indicated that GBs increased during ovulation, suggestive of enhanced reward sensitivity. Progesterone assays validated selfreported MCP (Study 2). Discussion and conclusions: The results suggest a role of ovarian hormones on negative affect and GBs in females. This research could lead to the identification of female-specific factors affecting gambling and the development of more effective interventions for females with, or at risk for, problematic gambling.
\end{abstract}

Keywords: gambling, alcohol consumption, menstrual cycle, female, self-medication, reward sensitivity

\section{INTRODUCTION}

Epidemiological studies suggest $65 \%-82 \%$ of the population has gambled on one or more occasion(s) in the past 12 months (Sproston, Hing, \& Palankay, 2012; Welte, Barnes, Wieczorek, Tidwell, \& Parker, 2002). Since gambling behavior (GB) is associated with social, psychologi$\mathrm{cal}$, and financial harms, it is important to identify factors contributing to increased GB.

Sex differences exist in GB. While males gamble at higher rates than females (Welte, Barnes, Tidwell, Hoffman, $\&$ Wieczorek, 2015), females begin gambling later (34.2 vs. 20.4 years old, respectively; Tavares, Zilberman, Beites, \& Gentil, 2001), prefer different types of gambling (Hraba \& Lee, 1996), have higher rates of comorbid mood/anxiety disorders (Ibáñez, Blanco, Moreryra, \& Saiz-Ruiz, 2003; Potenza et al., 2001), and are more likely to gamble to escape/relieve negative mood (Stewart \& Zack, 2008). Extant gambling research has focused on males. This sex insensitivity is problematic for at least two reasons. First, recent reports indicate an increased prevalence of GBs in females, with rates approaching those of males (RichmondRakerd, Slutske, \& Piasecki, 2013). Second, interventions

\footnotetext{
* Corresponding author: Dr. Sherry H. Stewart; Departments of Psychiatry and Psychology and Neuroscience, Dalhousie University, Life Sciences Centre, 1355 Oxford Street, Halifax NS, PO Box 15000, B3H 4R2, Canada; Phone: +1 902494 3793; Fax: +1 902 494 6585; E-mail: sstewart@dal.ca
}

This is an open-access article distributed under the terms of the Creative Commons Attribution-NonCommercial 4.0 International License, which permits unrestricted use, distribution, and reproduction in any medium for non-commercial purposes, provided the original author and source are credited, a link to the CC License is provided, and changes - if any - are indicated. 
targeting excessive gambling, stemming from this previous work, may not generalize to females.

One factor potentially influencing females' addictive behaviors is ovarian hormone fluctuations across the menstrual cycle (MC; Moran-Santa Maria, Flanagan, \& Brady, 2014; Terner \& de Wit, 2006). The average MC lasts 23-35 days (Münster, Schmidt, \& Helm, 1992), and is subdivided into the: menstrual (days 1-5), follicular (days 6-12), ovulatory (days 13-16), luteal (days 17 to premenstrual phase), and premenstrual phases ( 5 days prior to menstruation; Evans, Haney, Levin, Foltin, \& Fischman, 1998; Johannes et al., 1995; Pastor \& Evans, 2003). Mood fluctuations across MC phase (MCP) are believed to relate to variations in progesterone and estrogen concentrations, although no single underlying mechanism has been identified. Elevated negative affect is reported premenstrually/menstrually (Richards, Rubinow, Daly, \& Schmidt, 2006) - MCPs characterized by low estrogen and precipitously decreasing progesterone levels, respectively (Griffin \& Ojeda, 2004).

Addictive behavior motivations are increasingly being viewed as having state-like features. Situational factors, like mood, predict addictive behavior motivations (Arbeau, Kuiken, \& Wild, 2011). Self-medication theory (SMT; Khantzian, 1997) posits that addictive behaviors serve to reduce/eliminate elevated negative affect. Consistent with SMT predictions, increased alcohol consumption menstrually was explained by elevated coping motives (Joyce, Hudson, et al., 2018). Since female gamblers gamble to cope more frequently than males (Stewart \& Zack, 2008), and if gambling motivation similarly has state-like properties, females may increase risky GBs premenstrually/ menstrually (vs. other MCPs) to self-medicate elevated negative affect (Richards et al., 2006). Increased risky GBs premenstrually/menstrually might include the use of alcohol while gambling, since problem gamblers have an increased risk for problematic drinking (Zimmerman, Chelminski, \& Young, 2006). Further examining negative affect and GB across MCP appears to be a critical step toward identifying female-specific gambling factors.

Although findings vary across studies and the addictive behavior examined, evidence suggests MCP influences substance use/other addictive behaviors. Across substance use behaviors, results have been inconsistent; however, for alcohol, cigarettes, and cannabis, the slight majority of findings support increased substance use premenstrually and/or menstrually relative to other MCP, consistent with SMT predictions (Carroll, Lustyk, \& Larimer, 2015; Joyce, Good, Tibbo, Brown, \& Stewart, 2018).

Two studies have examined GBs across MCP in female auction bidders (Chen, Katuščák, \& Ozdenoren, 2013; Pearson \& Schipper, 2013). These studies were longitudinal, in-laboratory studies assessing the amount of money spent bidding, and bidding profits obtained, across MCP. Normally cycling females bid higher and earned lower profits than the average male during all MCPs except during the follicular phase (Pearson \& Schipper, 2013), suggesting females engage in less risky GB during the follicular phase. The results provided partial support for SMT predictions (i.e., riskier GB premenstrually/menstrually than during the follicular phase). However, another study suggested that females bid more during the follicular phase compared to the male average and female cycle average (Chen et al., 2013), providing no support for SMT predictions. However, this study was conducted on females taking hormonal contraceptives, introducing interpretive problems since ovarian hormones would not vary naturally across MCP. Overall, MCP effects on GB are limited to two studies employing a single index of laboratory-based bidding, raising issues of generalizability to other forms of gambling and external validity outside of the laboratory. In addition, the active form of gambling examined (i.e., auction bidding) lacks ecological validity as females more commonly gravitate toward passive types of gambling, such as bingo and slot machines (Hraba \& Lee, 1996).

The current studies address the literature gap on whether GBs vary across females' MCP through the implementation of retrospective (Study 1) and prospective (Study 2) research designs examining three indices of risky GB. The retrospective design allowed for the inclusion of a larger sample size, reducing error variance, and increasing generalizability. The prospective design reduced memory biases (Goldstein et al., 2017) and allowed for the collection of negative affect data, and the biological confirmation of MCP (e.g., Joyce, Hudson, et al., 2018). However, because prospective daily diary methods are more demanding versus other methods, it can be more difficult to find participants willing to provide daily assessments, and data may suffer due to participant non-compliance. As a result, retrospective and prospective designs were employed to enhance rigor and generalizability.

Based on SMT predictions (Khantzian, 1997), and established increases in negative affect premenstrually/ menstrually (Richards et al., 2006), we predicted that female gamblers would spend more time and money gambling, and would display a higher probability of consuming alcohol while gambling, premenstrually/menstrually versus other MCPs (Studies 1 and 2). Moreover, based on prior research (e.g., Richards et al., 2006), we hypothesized females would report elevated negative affect premenstrually/menstrually versus other MCPs (Study 2). Finally, we predicted salivary progesterone levels would be higher during self-reported MC days 1-7 than self-reported MC days 18-24, thus validating self-reported MCP (Study 2).

\section{METHODS: STUDY 1}

\section{Participants}

Thirty-three normally cycling female gamblers $\left(M_{\text {age }}=29.9\right.$ years, $S D=10.1$ ) were recruited through advertisements (community, campus, and social media). On average, participants gambled 5.12 days $(S D=3.66)$ during the prior 30 days and reported moderate levels of gambling consequences on the Problem Gambling Severity Index (PGSI; Ferris \& Wynne, 2001; $M=5.91, S D=5.25$; scores ranged from 1 to 25$)$. The majority of participants were low risk $(n=17)$, followed by moderate risk $(n=8)$ and problem gamblers $(n=8)$. Females were excluded if they gambled less than twice in the past month, were not between 19 and 45 years old, were abstaining/trying to abstain from gambling, self-reported a severe mood or psychiatric disorder 
diagnosis (aside from premenstrual dysphoric disorder; PMDD), experienced interference with their MC [hormonal contraceptive use, recent/current pregnancy, plans of conceiving, or (post)menopausal], were not normally cycling, or had a MC length outside of the average 25-32 days.

\section{Measures}

Problem Gambling Severity Index (PGSI). The PGSI (Ferris \& Wynne, 2001) is a nine-item questionnaire describing participants' gambling severity. An example question includes "Have you bet more than you could really afford to lose?" rated on a 4-point scale from "never" (0) to "almost always" (3). Responses were summed. Total scores were interpreted using established cut-offs: $0=$ nonproblem, $1-4=$ low risk, 5-7=moderate risk, and $8+=$ problem gamblers. The PGSI has good internal consistency $(\alpha=.84)$, test-retest reliability over $3-4$ weeks $(r=.78)$, and convergent validity with Lesieur and Blume's (1987) South Oaks Gambling Screen (Ferris \& Wynne, 2001).

MC Questionnaire (MCQ). The MCQ is an eight-item, author-compiled questionnaire assessing pregnancy, contraceptive use, and MC regularity (e.g., "Are you currently trying to conceive?") to confirm participant eligibility. The most recent first day of menstruation was also reported via the item "Thinking back to your last menstrual period, on what date did your period begin? (Count the first day of blood flow as day one, not spotting)." A count forwardbackward method, based on an average 28-day cycle, identified the participant's MC day on the Gambling Timeline Followback (GTLFB). Retrospective recall of the first day of menstruation is an accurate indicator of $\mathrm{MC}$ day with $81 \%$ of females accurately recalling menstruation within 2 days (Wegienka \& Baird, 2005).

Gambling timeline followback (GTLFB). The GTLFB (Weinstock, Whalen, \& Meyers, 2004) is a retrospective tool assessing participants' GB 30 days prior. A calendar was labeled with significant occasions during the past 30 days, to act as memory anchors. For each gambling occasion during the prior 30 days, participants reported the amount of time ["How much time did you spend gambling (in minutes)?"] and money spent gambling ["How much money did you spend gambling (in Canadian dollars)?'], and whether alcoholic beverages were consumed while gambling ["How many drinks did you consume while gambling (number of standard drinks)?;" coded as yes/no for analysis purposes]. A standard drink was specified as: one beer; one cooler; one 4-oz. glass of wine; or one shot/mixed drink containing 1-oz. of hard liquor. The three questions asked have acceptable to excellent test-retest reliability across a 6-month period [i.e., time spent $(r=.79)$, money spent $(r=.96)$, and drinks consumed $(r=.94)]$ and good concurrent and discriminant validity (Weinstock et al., 2004).

\section{Procedure}

Study 1 data were obtained between October 2013 and May 2015. A telephone screening determined eligibility. Those eligible females were invited to an in-laboratory session where they completed the PGSI, MCQ, and GTLFB. Participants were compensated $\$ 40 / \mathrm{CDN}$.

\section{Statistical analysis}

Data were standardized to an average 28-day cycle using a phasic standardization procedure developed by Joyce and Stewart (in press). Gambling variables were then calculated as rates per phase to account for variations in MCP length [Variability in total MC length could not be accounted for in Study 1; this was a limitation of the retrospective design used. To account for this limitation in Study 2, MC length variability was accounted for by including a variable number of days in the luteal phase for females with cycles greater than 28 days (see Joyce \& Stewart, in press). For females with cycles less than 32 days, only days within a full MC were used (and the rest were excluded)]. Time and money spent gambling were calculated as totals per phase and divided by the number of days gambled during each phase. The number of days where alcohol was consumed while gambling per phase was divided by the number of days gambled per phase. Missing data was then imputed using multiple imputations and data analyses were conducted using SPSS (IBM Corp. Released 2015, IBM SPSS Statistics for Windows, version 23.0. Armonk, NY, USA). Composite variables were developed for each GB by collapsing participant data across MCP, providing a variable for the average time and money spent gambling, and the proportion of gambling occasions where alcohol was consumed, over the prior 30 days. Bivariate correlations were conducted to examine overlap between these three GBs, irrespective of MCP. For hypothesis tests involving all three risky GBs, sets of non-parametric Wilcoxon rank tests compared each GB across MCP. Parametric tests rely on large sample sizes, normal distributions, and are influenced by outliers; thus, non-parametric analyses were employed due to the study's small sample size.

\section{Ethics}

The procedures of Studies 1 and 2 abided by the Declaration of Helsinki. Studies 1 and 2 were approved by research ethics boards at Dalhousie University (Studies 1 and 2), the University of Toronto (Studies 1 and 2), Concordia University (Study 2), and the University of Manitoba (Study 1). All participants provided informed consent prior to participation.

\section{RESULTS}

Bivariate correlations, collapsed across MCP, indicated that the amount of time and money spent gambling were significantly intercorrelated. None of the remaining correlations were significant (Table 1). The general independence of the three dependent variables justified their examination as independent outcomes.

The total amount of time spent gambling per gambling occasion differed by MCP. Inconsistent with SMT predictions, females spent less time gambling per gambling occasion menstrually $(M d n=45.00$; range $=240.00)$ than during the follicular $(M d n=70.34$; range $=235.00)$, ovulatory $(M d n=104.58 ;$ range $=300.00)$, and luteal $(M d n=81.69$; range $=300.00)$ phases, and more time gambling per gambling occasion during ovulation than premenstrually 
Joyce et al.

Table 1. Summary of means, standard deviations, and intercorrelations for money and time spent gambling, the probability of consuming alcohol while gambling, and negative affect

\begin{tabular}{|c|c|c|c|c|c|c|c|}
\hline Measures & 1 & 2 & 3 & 4 & $M$ & $M d n$ & $S D$ \\
\hline 1. Money spent gambling (dollars) & - & $.375^{*}$ & -.105 & & 84.64 & 35.92 & 99.00 \\
\hline 2. Time spent gambling (minutes) & .223 & - & .077 & & 85.02 & 84.22 & 55.08 \\
\hline 3. Alcohol consumption while gambling & .081 & .184 & - & & .30 & .20 & .29 \\
\hline 4. Negative affect & -.210 & .032 & .071 & - & & & \\
\hline$M$ & 25.69 & 95.86 & .38 & 15.60 & & & \\
\hline$M d n$ & 24.55 & 74.14 & .41 & 10.76 & & & \\
\hline$S D$ & 12.13 & 103.91 & .18 & 14.10 & & & \\
\hline
\end{tabular}

Note. Intercorrelations for Study $1(n=33)$ are presented above the diagonal, and intercorrelations for Study $2(n=20)$ are presented below the diagonal. Means and standard deviations (SDs) for Study 1 are presented in the vertical columns, whereas the means and SDs for Study 2 are presented in the horizontal columns. Negative affect was not measured in Study 1; thus, correlations, mean, median, and standard deviations are not included. All tests were two-tailed.

*Significance at the $p<.05$ level.

(a)

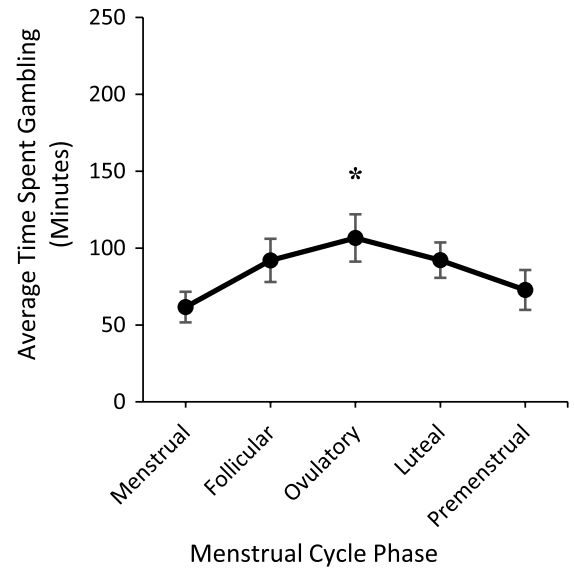

(b)

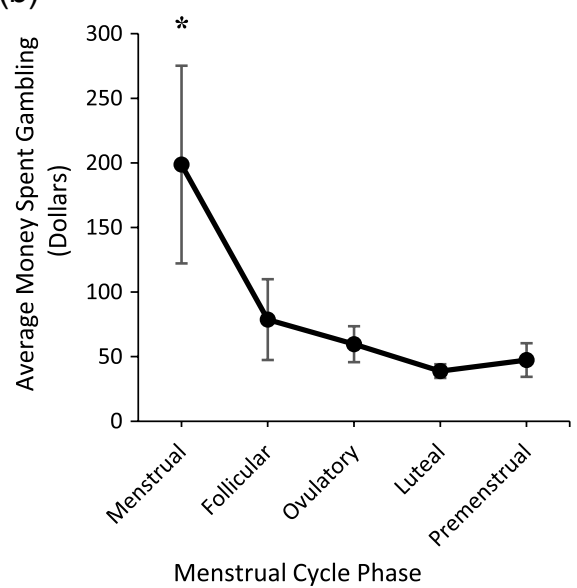

(c)

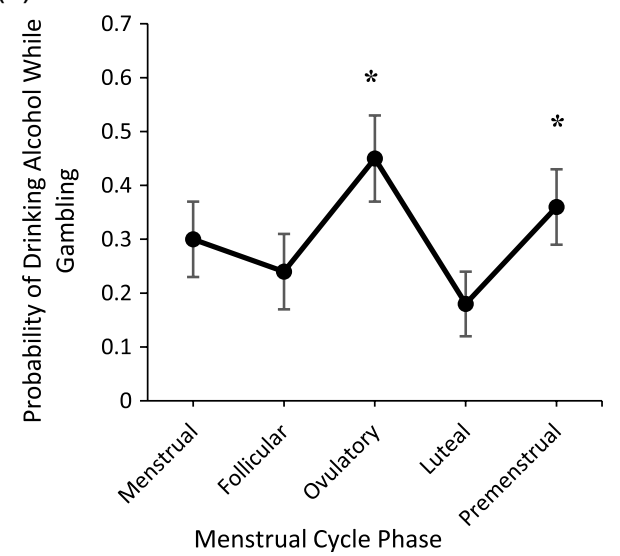

Figure 1. Average time (minutes) spent gambling per gambling occasion (a), money (Canadian dollars) spent gambling per gambling occasion (b), and probability of drinking while gambling (c) across menstrual cycle phase in retrospective Study 1. Error bars represent standard errors. Note. Asterisk $(*)$ indicates significantly higher levels of gambling behavior when compared to at least one other menstrual cycle phase

$(M d n=47.16 ;$ range $=299.00 ;$ Figure 1a; Table 2). The amount of money spent per gambling occasion did not differ based on MCP (Figure 1b; Table 2).

The probability of drinking alcohol while gambling differed by MCP. Partially consistent with SMT predictions, the probability of drinking alcohol while gambling was greater premenstrually $(M d n=0.33$; range $=1)$ than during the follicular $(M d n=0.00$; range $=1$; marginally significant) and luteal phases $(M d n=0.00 ;$ range $=1$; Figure 1c). The probability of consuming alcohol while gambling was also greater during ovulation $(M d n=0.39$; range $=1$ ) than all other MCPs (Figure 1c; Table 2). 
Table 2. Summary of Study 1 Wilcoxon rank tests of money spent gambling, time spent gambling, and alcohol consumed, while gambling across menstrual cycle phase pairs

\begin{tabular}{|c|c|c|c|c|}
\hline Variable & MCP pair & $Z$ & $p$ & $r$ \\
\hline \multirow[t]{10}{*}{ Dollars spent } & Menstrual-follicular & 0.745 & .456 & .130 \\
\hline & Menstrual-ovulatory & 0.831 & .406 & .145 \\
\hline & Menstrual-luteal & 0.168 & .866 & .029 \\
\hline & Menstrual-premenstrual & 0.333 & .736 & .058 \\
\hline & Follicular-ovulatory & 1.260 & .208 & .219 \\
\hline & Follicular-luteal & 0.049 & .961 & .009 \\
\hline & Follicular-premenstrual & 0.157 & .875 & .027 \\
\hline & Ovulatory-luteal & 0.785 & .432 & .137 \\
\hline & Ovulatory-premenstrual & 1.162 & .245 & .202 \\
\hline & Luteal-premenstrual & 0.524 & .601 & .091 \\
\hline \multirow[t]{10}{*}{ Alcohol consumed } & Menstrual-follicular & 0.871 & .384 & .151 \\
\hline & Menstrual-luteal & 1.856 & .063 & .323 \\
\hline & Menstrual-premenstrual & 0.532 & .595 & .092 \\
\hline & Follicular-luteal & 0.734 & .463 & .128 \\
\hline & Ovulatory-menstrual & 2.158 & $.031 *$ & .376 \\
\hline & Ovulatory-follicular & 2.653 & $.008^{*}$ & .462 \\
\hline & Ovulatory-luteal & 3.752 & $<.001 *$ & .653 \\
\hline & Ovulatory-premenstrual & 2.173 & $.030 *$ & .378 \\
\hline & Premenstrual-follicular & 1.960 & $.050 * *$ & .341 \\
\hline & Premenstrual-luteal & 2.596 & $.009 *$ & .452 \\
\hline \multirow[t]{10}{*}{ Time spent } & Menstrual-luteal & 2.515 & $.012 *$ & .438 \\
\hline & Menstrual-premenstrual & 0.720 & .472 & .125 \\
\hline & Follicular-menstrual & 2.440 & $.015^{*}$ & .425 \\
\hline & Follicular-ovulatory & 0.898 & .369 & .156 \\
\hline & Follicular-luteal & 0.206 & .837 & .036 \\
\hline & Follicular-premenstrual & 1.460 & .144 & .254 \\
\hline & Ovulatory-menstrual & 2.811 & $.005^{*}$ & .489 \\
\hline & Ovulatory-luteal & 0.972 & .331 & .169 \\
\hline & Ovulatory-premenstrual & 2.478 & $.013 *$ & .431 \\
\hline & Luteal-premenstrual & 1.284 & .199 & .224 \\
\hline
\end{tabular}

Note. All significant and marginally significant results are represented in bold. Directionality of each effect is shown in the "MC phase pair" column, with the highest value presented first. The column " $r$ " refers to the effect size of each phase comparison conducted (small effect size $=0.1$; medium effect size $=0.3$; large effect size $=0.5$ ). MCP: menstrual cycle phase.

*Significant differences at the $p<.05$ level. **Marginal significance at the $p<.06$ level.

\section{METHODS: STUDY 2}

\section{Participants}

Twenty normally cycling female gamblers $\left(M_{\text {age }}=30.7\right.$ years, $S D=9.48$ ) were recruited via the methods used in Study 1. On average, participants gambled 5.25 times $(S D=3.78)$ during their 32 days of study participation. Participants reported average MC lengths $(M=29.1$ days, $S D=3.74)$ and similar average PGSI scores $(M=4.60$, $S D=4.81$; scores ranged from 0 to 21 ) to Study 1 participants. The majority of participants were low risk $(n=12)$, followed by problem $(n=4)$, moderate risk $(n=3)$, and non-problem $(n=1)$ gamblers. Inclusion/exclusion criteria were identical to those in Study 1.

\section{Daily diary measures}

MC day. Participants identified their MC day daily, with day one being the first day of menstruation (i.e., "Please indicate your MC day, if known, with Day 1 being the first day of menstruation"). If $\mathrm{MC}$ day was unknown, participants indicated "unknown" until menstruation began. Reporting
MC day [vs. menstruation is occurring (yes/no)] is superior for determining MCP, as variable MC lengths are captured and errors determining the first day of menstruation are reduced (e.g., if a survey is missed; Joyce, Hudson, et al., 2018).

Gambling assessment. GB was measured prospectively, using questions identical to those on the retrospective GTLFB (Weinstock et al., 2004; Since GBs were reported for the previous day, a lag procedure was used for data scoring, where GBs were shifted back one day to align with the day during which the GBs occurred).

Negative affect assessment. State negative affect items were drawn from prior research (Grant, Stewart, \& Birch, 2007). Participants were presented with six negative affect adjectives (i.e., "sad," "depressed," "blue," "nervous," "anxious," and "tense"). The participant rated the extent to which she currently experienced each adjective using a Visual Analog Scale, with the scale ranging from "not at all" to "very." For scoring purposes, "not at all" was coded as zero; whereas "very" was coded as 100 , with possible scores ranging anywhere between these two values. Negative affect adjective scores were first averaged per day, producing an overall negative affect score per participant, 
per MC day. Phasic negative affect scores were calculated as totals per phase divided by the number of days within that MCP.

\section{Procedure}

Study 2 data were obtained between September 2016 and April 2017. Study 2 consisted of four phases: telephone screening, initial in-person assessment, 32 days of daily diary surveys and saliva collection in vivo, and an in-person debriefing session. The telephone screening determined eligibility. If eligible, the participant was invited to attend an initial assessment where she was instructed on how to complete daily diary surveys and collect saliva samples. Everyday for 32 days following the initial assessment, participants collected daily saliva samples and received daily diary text messages (at 10:30 a.m.), which provided links to a survey on the Internet. Due to variability in MC length, participants were monitored for 32 days, maximizing the likelihood of obtaining data across an entire MC (accommodating longer MC lengths). Participants were instructed to provide saliva samples simultaneously daily and to store samples in a freezer until returned. Once the daily surveys and saliva collection were completed, saliva samples were returned via a provided cooler. Proper storage was verified with participants. During debriefing, full information on the study's purpose was available and participants could ask questions. Participants were compensated up to $\$ 130 / C D N$. Compensation depended on how many daily surveys and saliva samples were provided to encourage compliance.

In the laboratory, saliva samples were stored in a research-grade freezer $\left(-30{ }^{\circ} \mathrm{C}\right)$ until progesterone assays were performed. The maximum period of storage was 8 months, as hormone concentrations remain stable without significant degradation during this period (Latendresse \& Ruiz, 2009). Salivary progesterone concentrations are non-invasive, reliable indicators of MCP designation (Andreano \& Cahill, 2010; Tallon et al., 1984). Salivary progesterone concentrations were assessed using two samples during MCPs of theoretical low (days 1-7) and high (days 18-24) progesterone concentrations (Andreano \& Cahill, 2010) using Salimetrics (State College, PA) ELISA kits.

\section{Statistical analysis}

The analytic strategy of Study 2 GBs was identical to that in Study 1 . In addition, internal consistency was calculated for the negative affect scores [Internal consistency between the six negative affect items were calculated at five time points across the MC. Self-reported negative affect item data was analyzed during the middle day of each MCP. In the case that the number of days within a given MCP were even (i.e., there was not a single middle MC day), scores for each negative affect item were consistently taken from the later of the two middle MC days. That is, during the ovulatory phase (consisting of MC days 13-16), negative affect item scores were analyzed for MC day 15] and Wilcoxon rank tests were used to compare negative affect across MCP. Negative affect and GB data were further collapsed across MCP, providing average self-reported negative mood and average GBs across the entire MC. Bivariate correlations were then calculated for all negative affect and risky GB variables to examine their overlap irrespective of MCP. Finally, a dependent sample $t$-test examined progesterone concentrations at two predetermined MCPs to validate self-reported MCP.

\section{RESULTS}

None of the correlations between the four dependent variables were significant when collapsed across MCP (Table 1). This independence of the dependent variables justified their examination as independent outcomes.

The amount of time spent gambling per gambling occasion differed by MCP. Partially consistent with SMT predictions, females spent more time gambling per gambling occasion menstrually $(M d n=60$; range $=220.00)$ than during the follicular phase $(M d n=42.54$; range $=87.50)$ and more time gambling per gambling occasion premenstrually $(M d n=103.08$; range $=925.00)$ than during the follicular, ovulatory $(M d n=69.46$; range $=137.50)$, and luteal phases $(M d n=68.36 ; \quad$ range $=160.00)$. Unexpectedly, females spent more time gambling per gambling occasion during ovulation than the follicular phase (Figure 2a; Table 3).

The amount of money spent gambling per gambling occasion differed by MCP. Partially consistent with SMT predictions, females spent more money per gambling occasion menstrually $(M d n=33.92$; range $=97.00)$ than during the follicular $(M d n=12.53$; range $=26.00)$, luteal $(M d n=$ 20.49 ; range $=72.00)$, and premenstrual phases $(M d n=$ 6.83; range $=73.00)$. However, in direct contrast to SMT predictions, less money was spent per gambling occasion premenstrually versus all other MCPs. Females also spent more money per gambling occasion during ovulation $(M d n=39.03$; range $=196.00)$ than the follicular, luteal, and premenstrual phases (Figure 2b; Table 3).

The probability of drinking alcohol while gambling differed based on MCP. In contrast to SMT predictions, the probability of drinking alcohol while gambling was greater during ovulation $(M d n=0.615$; range $=1)$ than the menstrual $(M d n=0.302 ;$ range $=1)$, follicular $(M d n=$ 0.205 ; range $=1)$, and premenstrual phases $(M d n=0.325$; range $=1$; Figure 2c; Table 3 ).

Internal consistency for the six negative affect items ranged from acceptable $(\alpha=.774)$ to excellent $(\alpha=.938)$. Negative affect differed based on MCP. Consistent with SMT predictions, negative affect was greater premenstrually $(M d n=14.28$; range $=57.23)$ than during the luteal phase $(M d n=7.55$; range $=41.33)$ and marginally greater menstrually $(M d n=10.67$; range $=59.28)$ than during ovulation $(M d n=6.05$; range $=49.00$; Figure 2d; Table 3$)$.

\section{Progesterone assays}

For Study 2, progesterone assays were conducted using two samples per MCP of interest per participant. The first sample assayed was where, according to self-reported MC day, progesterone concentrations should have been highest (days 18-24) or lowest (days 1-7; Andreano \& Cahill, 2010). 
(a)

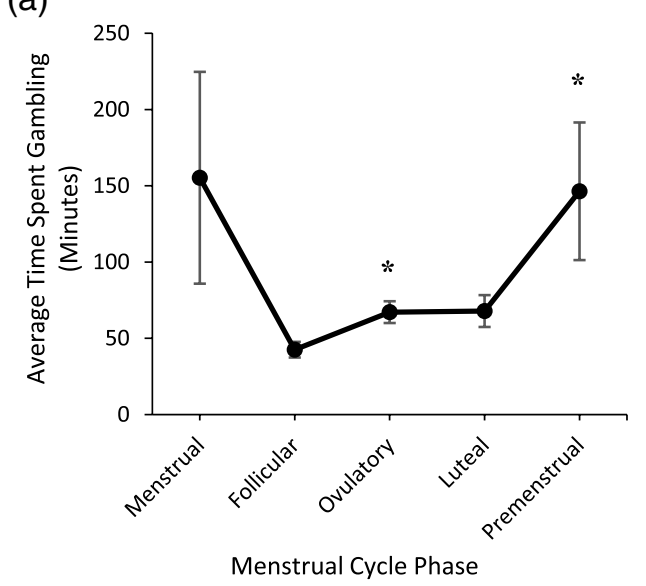

(c)

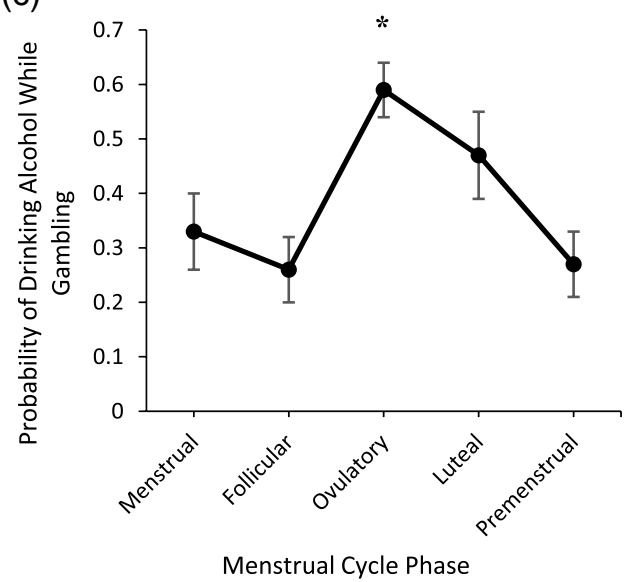

(b)

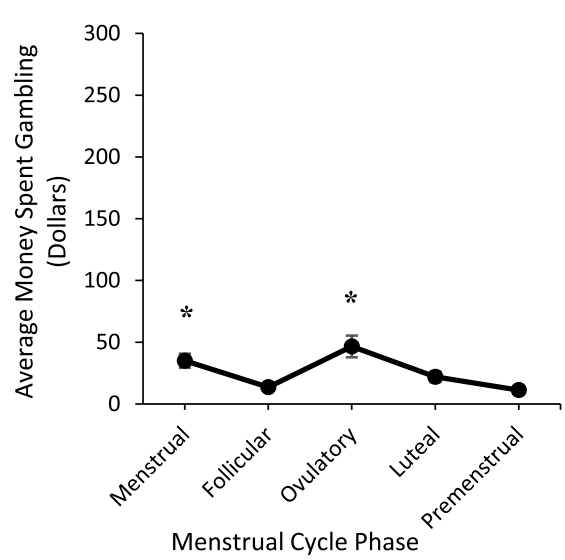

(d)

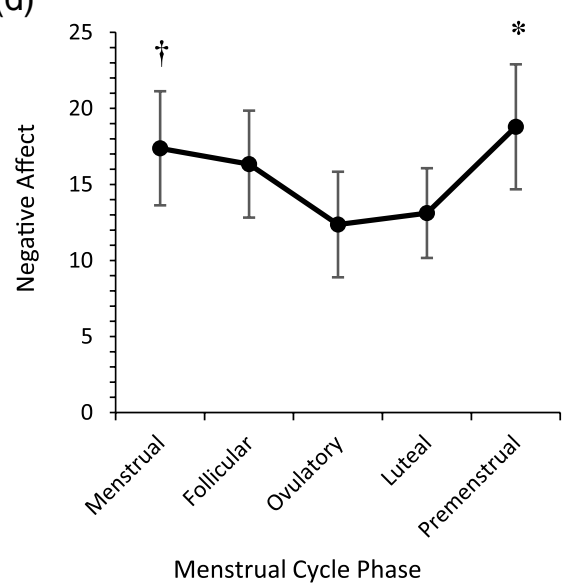

Figure 2. Average time (minutes) spent gambling per gambling occasion (a), money (Canadian dollars) spent gambling per gambling occasion (b), the probability of drinking while gambling (c), and negative affect (d) across menstrual cycle phase in the prospective Study 2. Error bars represent standard errors. Note. Asterisk $\left(^{*}\right)$ indicates significantly higher levels and a cross symbol ( $\dagger$ ) indicates marginally significant higher levels of gambling behavior/negative affect relative to at least one other menstrual cycle phase

The second sample was from 1 day before the first sample $(n=10)$, or in the case of unavailable/contaminated samples, the closest sample to the first sample $(n=4$; all within 2 days of the empty/spoiled sample) [Progesterone concentration assays were completed for $70 \%$ of participants (14 out of 20) as four participants (20\%) did not return their saliva samples following study completion, and progesterone concentrations were outside of the detectable range for two participants $(10 \%)$ likely due to improper storage]. Within both phases, the two samples were highly correlated $[r$ 's $=.88$ (days 1-7), and .83 (days 18-24)], so they were averaged within phase. Averaged values showed a MCP effect $[t(13)=2.82, p=.014]$ with progesterone concentrations significantly higher, on average, during days $18-24$ than days $1-7$ [M's $(S E$ 's $)=135.13(38.38)$ vs. 64.51 (21.06) $\mathrm{pg} / \mathrm{ml}$, respectively], as expected, providing validation for self-reported MCP.

\section{DISCUSSION}

The multimethod approach used here provided the first thorough examination of differences in various GBs across female gamblers' MCs. Based on SMT (Khantzian, 1997), we hypothesized female gamblers would engage in riskier GBs and experience higher levels of negative affect premenstrually/menstrually versus other MCPs. Findings provided partial support for SMT predictions. Unexpectedly, the results more consistently suggested female gamblers engage in riskier GBs during ovulation.

Both studies partially supported SMT predictions. Study 1 found an increased probability of consuming alcohol while gambling premenstrually [relative to the luteal and follicular (marginal significance) phases]. However, these effects were not replicated in prospective Study 2. In addition, Study 2 found increases in the amount of time spent gambling menstrually and premenstrually (relative to the follicular phase, and to the follicular, ovulatory, and luteal phases, respectively). However, these effects were not observed in retrospective Study 1. Study 2 also found increases in the amount of money spent gambling menstrually (relative to the follicular, luteal, and premenstrual phases), but again this effect was not observed in Study 1. In direct contrast to SMT predictions, however, Study 1 found that time spent gambling per gambling occasion was lower menstrually (relative to the follicular, ovulatory, and luteal phases) and Study 2 found the amount of money spent gambling was lowest 
Table 3. Summary of Study 2 Wilcoxon rank tests of money spent gambling, time spent gambling, alcohol consumed while gambling, and negative affect across menstrual cycle phase pairs

\begin{tabular}{|c|c|c|c|c|}
\hline Variable & MCP pair & $Z$ & $p$ & $r$ \\
\hline \multirow[t]{10}{*}{ Dollars spent } & Menstrual-follicular & 3.340 & $.001 *$ & .747 \\
\hline & Menstrual-luteal & 2.439 & $.015 *$ & .545 \\
\hline & Menstrual-premenstrual & 3.136 & $.002 *$ & .701 \\
\hline & Follicular-premenstrual & 2.052 & $.040 *$ & .459 \\
\hline & Ovulatory-menstrual & 1.643 & .100 & .367 \\
\hline & Ovulatory-follicular & 3.659 & $<.001 *$ & .818 \\
\hline & Ovulatory-luteal & 3.114 & $.002 *$ & .696 \\
\hline & Ovulatory-premenstrual & 3.211 & $.001 *$ & .718 \\
\hline & Luteal-follicular & 1.808 & .071 & .404 \\
\hline & Luteal-premenstrual & 2.455 & $.014 *$ & .549 \\
\hline \multirow[t]{10}{*}{ Alcohol consumed } & Menstrual-follicular & 0.966 & .334 & .216 \\
\hline & Ovulatory-menstrual & 2.722 & $.006^{*}$ & .609 \\
\hline & Ovulatory-follicular & 3.245 & $.001 *$ & .726 \\
\hline & Ovulatory-luteal & 1.208 & .227 & .270 \\
\hline & Ovulatory-premenstrual & 3.527 & $<.001 *$ & .789 \\
\hline & Luteal-menstrual & 1.294 & .196 & .289 \\
\hline & Luteal-follicular & 2.172 & $.030 *$ & .486 \\
\hline & Luteal-premenstrual & 2.274 & $.023 *$ & .508 \\
\hline & Premenstrual-menstrual & 1.061 & .289 & .237 \\
\hline & Premenstrual-follicular & 0.345 & .730 & .077 \\
\hline \multirow[t]{10}{*}{ Time spent } & Menstrual-follicular & 2.501 & $.012 *$ & .561 \\
\hline & Ovulatory-menstrual & 1.415 & .157 & .316 \\
\hline & Ovulatory-follicular & 2.613 & $.009 *$ & .584 \\
\hline & Ovulatory-luteal & 0.762 & .446 & .170 \\
\hline & Luteal-menstrual & 1.248 & .212 & .279 \\
\hline & Luteal-follicular & 2.367 & $.018 *$ & .529 \\
\hline & Premenstrual-menstrual & 1.269 & .204 & .284 \\
\hline & Premenstrual-follicular & 3.361 & $.001 *$ & .752 \\
\hline & Premenstrual-ovulatory & 2.314 & $.021 *$ & .517 \\
\hline & Premenstrual-luteal & 2.173 & $.030 *$ & .486 \\
\hline \multirow[t]{10}{*}{ Negative affect } & Menstrual-ovulatory & 1.941 & $.052 * *$ & .434 \\
\hline & Menstrual-luteal & 1.755 & .079 & .392 \\
\hline & Follicular-menstrual & 0.485 & .627 & .108 \\
\hline & Follicular-ovulatory & 0.966 & .334 & .216 \\
\hline & Follicular-luteal & 1.493 & .135 & .334 \\
\hline & Luteal-ovulatory & 0.196 & .845 & .044 \\
\hline & Premenstrual-menstrual & 0.597 & .940 & .133 \\
\hline & Premenstrual-follicular & 0.635 & .526 & .142 \\
\hline & Premenstrual-ovulatory & 1.792 & .073 & .401 \\
\hline & Premenstrual-luteal & 1.979 & $.048 *$ & .443 \\
\hline
\end{tabular}

Note. All significant and marginally significant results are represented in bold. Directionality of each effect is shown in the "MCP pair" column, with the highest value presented first. The column " $r$ " refers to the effect size of each phase comparison conducted (small effect size $=0.1$; medium effect size $=0.3$; large effect size $=0.5$ ). MCP: menstrual cycle phase.

*Significant differences between menstrual cycle phase at the $p<.05$ level. **Marginal significance at the $p<.06$ level.

premenstrually (relative to all other MCP) and the probability of consuming alcohol while gambling was lower menstrually and premenstrually (relative to the ovulatory phase).

SMT predictions (Khantzian, 1997) were based on established increases in depressed mood premenstrually/ menstrually (Richards et al., 2006). Consistent with this prior work, we showed that negative affect increased premenstrually (relative to the luteal phase) and marginally increased menstrually (relative to the ovulatory phase). This shows that it was not the absence of expected negative affect changes across MCP explaining this failure to consistently obtain expected increases in GB menstrually/ premenstrually. It is plausible that GBs increase premenstrually/menstrually only for a subset of female gamblers who experience severely increased dysphoria premenstrually/ menstrually (e.g., those with PMDD; see DSM-5, American Psychiatric Association, 2013). Unfortunately, our small sample in Study 2 and lack of negative affect data in Study 1 precluded an examination of this possibility in our data. Thus, future research could compare GB findings across the MC for females with high versus low levels of PMDD symptoms to test whether SMT is more relevant for female gamblers with high PMDD with respect to GB 
across MCP. Future research could also investigate whether SMT predictions are borne out more consistently for female gamblers with diagnosed mood disorders (e.g., depression and anxiety). Herein, participants self-reporting a mood or psychiatric disorder diagnosis were excluded. Incorporating diagnostic measures, such as the Structured Clinical Interview for DSM-5 Disorders (First, Williams, Karg, \& Spitzer, 2015), to identify females with mood/anxiety disorders to ensure more rigorous assessment of the presence of these psychopathologies may be beneficial.

Since depressed individuals are more likely to report wanting to be secluded from others (Larson, Raffaelli, Richards, Ham, \& Jewell, 1990), females with significant PMDD symptomatology may engage in more solitary GB to self-medicate premenstrually/menstrually relative to female gamblers without high PMDD symptomatology. Future research should examine this, as the association between MCP and social gambling context (solitary vs. social; Quinlan, Goldstein, \& Stewart, 2013) was not explored in the current studies.

Unexpectedly, findings more consistently suggested risky GBs increased during ovulation versus at least one other MCP. This increase in risky GBs during ovulation may be attributable to fluctuations in ovarian hormones and/ or variations in positive mood across MCP. The surge in estrogen occurring during ovulation produces elevated sensitivity to rewards (Dreher et al., 2007; Sakaki \& Mather, 2012). Thus, female gamblers may increase their risky GBs to optimize perceived gambling-related rewards. Females also report their most positive mood during ovulation (Collins, Eneroth, \& Landgren, 1985); thus, it is possible that increased positive mood during ovulation prompts increased enhancement-motivated gambling to maximize positive mood experiences. Others should examine whether fluctuations in GB across MCP are mediated by changes in: gambling motives (state enhancement and/or coping; see Joyce, Hudson, et al., 2018, for a similar approach used for alcohol), and/or ovarian hormone concentrations (collected via daily hormonal assessments). Although these ovulatory phase-related findings are informative, they should be interpreted with caution as the present studies were not designed to detect the precise timing of ovulation. Future research should assess luteinizing hormone concentrations daily to more accurately pinpoint ovulation (Nardelli-Haefliger et al., 2003).

Findings could be used as a psychoeducational tool for female gamblers and to inform interventions for females experiencing, or at risk for, comorbid gambling and alcohol use problems. Providing information on the relationship between MCP and risky GBs could enhance awareness of MCPs where GBs may increase, allowing for the implementation of harm-reduction strategies to minimize GBs at riskier phases. Findings emphasize how using strategies to manage increases in reward sensitivity during ovulation may be beneficial upon implementing gambling interventions. If abstaining from gambling is the goal of treatment, initiating cessation attempts at lower-risk MCPs may enhance the likelihood of success; similar recommendations have been made for tobacco cessation based on MCP variability in smoking behavior (Mendrek, Dinh-Williams, Bourque, \& Potvin, 2014).
This study provides the first multimethod approach examining various GBs across an entire MC. Support for SMT was not consistent across retrospective and prospective methodologies, in which GBs were sometimes, but not always, found to increase premenstrually/menstrually versus another $\mathrm{MCP}(\mathrm{s})$. Unexpectedly, GBs quite consistently increased during ovulation. When results conflict between studies, we recommend emphasizing the prospective Study 2 results, given their reduced reliance on retrospective memory, our simultaneous assessment of negative affect, and our ability to validate MCP via progesterone assays. The results bring into question the reliability of employing retrospective methodologies in this area of research. Findings provide researchers with a more comprehensive understanding of how MCP influences females' GB and could aid in the development of more effective female-specific gambling harm-reduction tools.

Funding sources: The two studies presented herein were funded by an operating grant from the Manitoba Gambling Research Program (MGRP) awarded to Drs. SHS, ME, and ALG. The findings and conclusions of this paper are those solely of the authors and do not necessarily represent the views of Manitoba Lotteries. During this research, Ms. KMJ was supported by a Summer Studentship from the Dalhousie University Department of Psychiatry and her graduate studies in psychiatry research were supported by a Nova Scotia Graduate Scholarship, a Scotia Scholar Award from the Nova Scotia Health Research Foundation (NSHRF), and a Joseph Armand Bombardier Canada Graduate Scholarship from the Social Sciences and Humanities Research Council of Canada (SSHRC), Dr. AH was supported by a CIHR IMPART postdoctoral fellowship in Gender and Addictions, Dr. RMO'C by a CIHR New Investigator Award, Dr. ALG by the Canada Research Chairs Program, Dr. DSM by a Research Chair from the Alberta Gambling Research Institute, Dr. TSP by a NSERC Discovery Grant, and Dr. SHS by a CIHR Tier 1 Canada Research Chair in Addictions and Mental Health at Dalhousie University.

Authors' contribution: Ms. KMJ was responsible for developing the concepts and designs for Studies 1 and 2, recruiting participants, analysis and interpretation of data, and manuscript development. Dr. AH was responsible for developing the concepts and designs and co-supervised Ms. KMJ during the project. Dr. RMO'C was responsible for recruiting participants for Study 2 and co-supervised Dr. AH while completing her postdoctoral fellowship. Dr. ALG was involved in obtaining funding and recruiting participants for Studies 1 and 2. Dr. ME was involved in obtaining funding and recruiting participants for Study 1 . Dr. DSM was involved in developing the concepts and designs for Studies 1 and 2. Dr. TSP was responsible for conducting the progesterone assays for Study 2. Finally, Dr. SHS was responsible for obtaining funding, developing the concepts and designs for Studies 1 and 2, recruiting participants, data analysis and interpretation, and the cosupervision of Ms. KMJ during this project. All authors were responsible for revising and approving the manuscript prior to submission. 
Conflict of interest: The authors declare no conflict of interest.

Acknowledgements: The authors would like to acknowledge Pam Collins, Mayesha Khan, Laura MacKew, Charlotte Corran, and Ainsley Cloutier for assistance in data collection. They would also like to thank Elizabeth O'Leary for assistance in conducting the progesterone assays.

\section{REFERENCES}

American Psychiatric Association. (2013). Diagnostic and statistical manual of mental disorders (5th ed.). Washington, DC: American Psychiatric Association.

Andreano, J. M., \& Cahill, L. (2010). Menstrual cycle modulation of medial temporal activity evoked by negative emotion. Neuroimage, 53(4), 1286-1293. doi:10.1016/j.neuroimage. 2010.07.011

Arbeau, K. J., Kuiken, D., \& Wild, T. C. (2011). Drinking to enhance and to cope: A daily process study of motive specificity. Addictive Behaviors, 36(12), 1174-1183. doi:10.1016/j. addbeh.2011.07.020

Carroll, H. A., Lustyk, K. B., \& Larimer, M. E. (2015). The relationship between alcohol consumption and menstrual cycle: A review of the literature. Archives of Women's Mental Health, 18(6), 773-781. doi:10.1007/s00737-015-0568-2

Chen, Y., Katuščák, P., \& Ozdenoren, E. (2013). Why can’t a woman bid more like a man? Games and Economic Behavior, 77(1), 181-213. doi:10.1016/j.geb.2012.10.002

Collins, A., Eneroth, P., \& Landgren, B. M. (1985). Psychoneuroendocrine stress responses and mood as related to the menstrual cycle. Psychosomatic Medicine, 47(6), 512-527. doi:10.1097/00006842-198511000-00002

Dreher, J. C., Schmidt, P. J., Kohn, P., Furman, D., Rubinow, D., \& Berman, K. F. (2007). Menstrual cycle phase modulates reward-related neural function in women. Proceedings of the National Academy of Sciences of the United States of America, 104(7), 2465-2470. doi:10.1073/pnas.0605569104

Evans, S. M., Haney, M., Levin, F. R., Foltin, R. W., \& Fischman, M. W. (1998). Mood and performance changes in women with premenstrual dysphoric disorder: Acute effects of alprazolam. Neuropsychopharmacology, 19(6), 499-516. doi:10.1016/ S0893-133X(98)00064-5

Ferris, J., \& Wynne, H. J. (2001). The Canadian Problem Gambling Index: Final report. Ottawa, ON: Canadian Centre of Substance Abuse.

First, M., Williams, J., Karg, R., \& Spitzer, R. (2015). Structured Clinical Interview for DSM-5 diagnosis - Research version (SCID-5 for DSM-5 research version; SCID-5 RV). Arlington, VA: American Psychiatric Association.

Goldstein, A. L., Vilhena-Churchill, N., Munroe, M., Stewart, S. H., Flett, G. L., \& Hoaken, P. N. S. (2017). Understanding the effects of social desirability on gambling self-reports. International Journal of Mental Health and Addiction, 15(6), 1342-1359. doi:10.1007/s11469-016-9668-0

Grant, V. V., Stewart, S. H., \& Birch, C. D. (2007). Impact of positive and anxious mood on implicit alcohol-related cognitions in internally-motivated undergraduate drinkers.
Addictive Behaviors, 32(10), 2226-2237. doi:10.1016/j.addbeh. 2007.02.012

Griffin, J. E., \& Ojeda, S. R. (2004). Textbook of endocrine physiology. New York, NY: Oxford University Press.

Hraba, J., \& Lee, G. (1996). Gender, gambling and problem gambling. Journal of Gambling Studies, 12(1), 83-101. doi:10.1007/BF01533191

Ibáñez, A., Blanco, C., Moreryra, P., \& Saiz-Ruiz, J. (2003). Gender differences in pathological gambling. Journal of Clinical Psychiatry, 64(3), 295-301. doi:10.4088/JCP.v64n0311

Johannes, C. B., Linet, M. S., Stewart, W. F., Celentano, D. D., Lipton, R. B., \& Szklo, M. (1995). Relationship of headache to phase of the menstrual cycle among young women: A daily diary study. Neurology, 45(6), 1076-1082. doi:10.1212/WNL. 45.6.1076

Joyce, K. M., Good, K., Tibbo, P., Brown, J., \& Stewart, S. H. (2018, November). Addictive behaviors across the menstrual cycle: A systematic review. Poster presented at the Canadian Research Institute in Substance Misuse symposium, Moncton, NB.

Joyce, K. M., Hudson, A., O’Connor, R. M., Thompson, K., Hodgin, M., Perrot, T., \& Stewart, S. H. (2018). Changes in coping and social motives for drinking and alcohol consumption across the menstrual cycle. Depression and Anxiety, 35(4), 313-320. doi:10.1002/da.22699

Joyce, K. M., \& Stewart, S. H. (in press). Standardization of menstrual cycle data for the analysis of intensive longitudinal data. In O. I. Lutsenk (Ed.), Menstrual cycle. London, UK: Intech Open Science.

Khantzian, E. J. (1997). The self-medication hypothesis of substance use disorders: A reconsideration and recent applications. Harvard Review of Psychiatry, 4(5), 231-244. doi:10.3109/ 10673229709030550

Larson, R. W., Raffaelli, M., Richards, M. H., Ham, M., \& Jewell, L. (1990). Ecology of depression in late childhood and early adolescence: A profile of daily states and activities. Journal of Abnormal Psychology, 99(1), 92-102. doi:10.1037/0021843X.99.1.92

Latendresse, G., \& Ruiz, R. J. (2009). Bioasssay research methodology: Measure CRH in pregnancy. Biological Research for Nursing, 10(1), 54-62. doi:10.1177/1099800408320970

Lesieur, H. R., \& Blume, S. B. (1987). The South Oaks Gambling Screen (SOGS): A new instrument for the identification of pathological gamblers. American Journal of Psychiatry, 144(9), 1184-1188. doi:10.1176/ajp.144.9.1184

Mendrek, A., Dinh-Williams, L., Bourque, J., \& Potvin, S. (2014). Sex differences and menstrual cycle phase-dependent modulation of craving for cigarette: An fMRI pilot study. Psychiatry Journal, 2014, 1-7. doi:10.1155/2014/723632

Moran-Santa Maria, M. M., Flanagan, J., \& Brady, K. (2014). Ovarian hormones and drug abuse. Current Psychiatry Reports, 16(11), 511. doi:10.1007/s11920-014-0511-7

Münster, K., Schmidt, L., \& Helm, P. (1992). Length and variation in the menstrual cycle: A cross-sectional study from a Danish county. British Journal of Obstetrics and Gynaecology, 99(5), 422-429. doi:10.1111/j.1471-0528.1992.tb13762.x

Nardelli-Haefliger, D., Wirthner, D., Schiller, J. T., Lowy, D. R., Hildesheim, A., Ponci, F., \& De Grandi, P. (2003). Specific antibody levels at the cervix during the menstrual cycle of women vaccinated with human papillomavirus 16 virus-like 
particles. Journal of the National Cancer Institute, 95(15), 1128-1137. doi:10.1093/jnci/djg018

Pastor, A. D., \& Evans, S. M. (2003). Alcohol outcome expectancies and risk for alcohol use problems in women with and without a family history of alcoholism. Drug and Alcohol Dependence, 70(2), 201-214. doi:10.1016/S0376-8716(03) 00007-3

Pearson, M., \& Schipper, B. C. (2013). Menstrual cycle and competitive bidding. Games and Economic Behaviour, 78, 1-20. doi:10.1016/j.geb.2012.10.008

Potenza, M. N., Steinberg, M. A., McLaughlin, S. D., Wu, R., Rounsaville, B. J., \& O’Malley, S. S. (2001). Gender-related differences in the characteristics of problem gamblers using a gambling help line. American Journal of Psychiatry, 158(9), 1500-1505. doi:10.1176/appi.ajp.158.9.1500

Quinlan, C. K., Goldstein, A. L., \& Stewart, S. H. (2013). An investigation of the link between gambling motives and social context of gambling in young adults. International Gambling Studies, 14(1), 115-131. doi:10.1080/14459795.2013.855252

Richards, M., Rubinow, D. R., Daly, R. C., \& Schmidt, P. J. (2006). Premenstrual symptoms and perimenopausal depression. American Journal of Psychiatry, 163(1), 133-137. doi:10.1176/appi.ajp.163.1.133

Richmond-Rakerd, L. S., Slutske, W. S., \& Piasecki, T. M. (2013). Birth cohort and sex differences in the age of gambling initiation in the United States: Evidence from the national comorbidity survey replication. International Gambling Studies, 13(3), 417-429. doi:10.1080/14459795.2013.836554

Sakaki, M., \& Mather, M. (2012). How reward and emotional stimuli induce different reactions across the menstrual cycle. Social and Personality Psychology Compass, 6(1), 1-17. doi:10.1111/j.1751-9004.2011.00415.x

Sproston, K., Hing, N., \& Palankay, C. (2012). Prevalence of gambling and problem gambling in New South Wales. Sydney, Australia: New South Wales Office of Liquor, Gambling, \& Racing.
Stewart, S. H., \& Zack, M. (2008). Development and psychometric evaluation of a Three-Dimensional Gambling Motives Questionnaire. Addiction, 103(7), 1110-1117. doi:10.1111/j.13600443.2008.02235.x

Tallon, D. F., Gosling, J. P., Buckley, P. M., Dooley, M. M., Cleere, W. F., O’Dwyer, E. M., \& Fottrell, P. F. (1984). Direct solid-phase enzyme immunoassay of progesterone in saliva. Clinical Chemistry, 30, 1507-1511.

Tavares, H., Zilberman, M. L., Beites, F. J., \& Gentil, V. (2001). Gender differences in gambling progression. Journal of Gambling Studies, 17(2), 151-159. doi:10.1023/A:1016620513381

Terner, J. M., \& de Wit, H. (2006). Menstrual cycle phase and responses to drugs of abuse in humans. Drug and Alcohol Dependence, 84(1), 1-13. doi:10.1016/j.drugalcdep.2005. 12.007

Wegienka, G., \& Baird, D. D. (2005). A comparison of recalled date of last menstrual period with prospectively recorded dates. Journal of Women's Health, 14(3), 248-252. doi:10.1089/ jwh.2005.14.248

Weinstock, J., Whelan, J. P., \& Meyers, A. W. (2004). Behavioural assessment of gambling: An application of the timeline followback method. Psychological Assessment, 16(1), 72-80. doi:10.1037/1040-3590.16.1.72

Welte, J. W., Barnes, G. M., Tidwell, M. C., Hoffman, J. H., \& Wieczorek, W. F. (2015). Gambling and problem gambling in the United States: Changes between 1999 and 2013. Journal of Gambling Studies, 31(3), 695-715. doi:10.1007/s10899-0149471-4

Welte, J. W., Barnes, G. M., Wieczorek, W. F., Tidwell, M. C., \& Parker, J. (2002). Gambling participation in the US: Results from a national survey. Journal of Gambling Studies, 18(4), 313-337. doi:10.1023/A:1021019915591

Zimmerman, M., Chelminski, I., \& Young, D. (2006). Prevalence and diagnostic correlates of DSM-IV pathological gambling in psychiatric outpatients. Journal of Gambling Studies, 22(2), 255-262. doi:10.1007/s10899-006-9014-8 